\title{
Three-Component Coupling Reactions of Thioform- amides with Organolithium and Grignard Reagents
}

Key words

three-component reaction

coupling reaction

thioformamides

organolithium

reagents

Grignard reagents

tertiary amines

thiolating agents

diarylmethylpiperazines

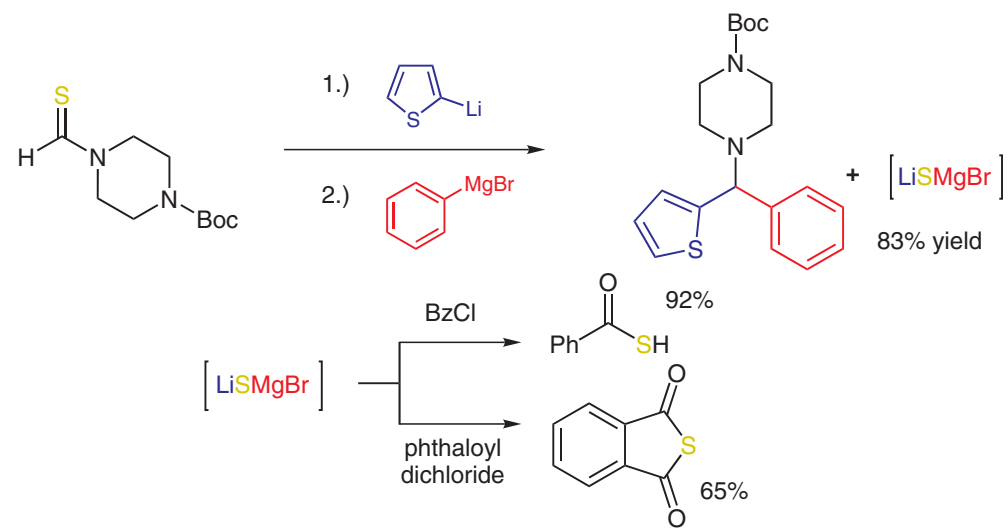

Significance: The use of $N, N$-dialkylthioformamides for the generation of tertiary amines via a novel three-component coupling reaction with organolithium and Grignard reagents is demonstrated. Thus, a variety of valuable tertiary amines, including the biologically significant diarylmethylpiperazines, were synthesized. It is noteworthy that the order of addition of the organolithium and Grignard reagents is vital to the success of the reaction. Remarkably, an excess of the organolithium reagent, which is first added to the $N, N$-dialkylthioformamide, does not interfere with the outcome of the reaction. In addition to the formation of tertiary amines a new thiolating agent, supposedly $\mathrm{LiSMgBr}$, is formed during the coupling reaction.
Comment: This three-component coupling reaction represents a new practical method for the synthesis of tertiary amines, as the combination of versatile organolithium and Grignard reagents allows for a large diversity of possible products. This method is also potentially relevant for medicinal chemistry, since racemic key intermediates of biologically active compounds, such as cetilidine, a $\mathrm{H} 1$-receptor agonist and rivastigmine, a drug administered for the treatment of Alzheimer's disease, can be easily accessed by it. The synthetic scope of the in situ generated thiolating agent has yet to be determined. 